\title{
Article \\ Biophysical and Biomechanical Effect of Low Intensity US Treatments on Pancreatic Adenocarcinoma 3D Cultures
}

\author{
Mattia Dimitri ${ }^{1,+}{ }^{\mathbb{D}}$, Claudia Duranti ${ }^{2,+}{ }^{+}$, Sara Aquino ${ }^{1} \mathbb{D}$, Lucrezia Mazzantini $^{1}$, Jessica Iorio ${ }^{2}$, Matteo Lulli ${ }^{3} \mathbb{D}$, \\ Martina Ricci ${ }^{1}$, Lorenzo Capineri ${ }^{4}$, Annarosa Arcangeli ${ }^{2, *, \ddagger}$ and Andrea Corvi ${ }^{1, \ddagger}$ (iD
}

check for

updates

Citation: Dimitri, M.; Duranti, C.; Aquino, S.; Mazzantini, L.; Iorio, J.; Lulli, M.; Ricci, M.; Capineri, L.; Arcangeli, A.; Corvi, A. Biophysical and Biomechanical Effect of Low Intensity US Treatments on Pancreatic Adenocarcinoma 3D Cultures. Appl. Sci. 2022, 12, 666. https://doi.org/10.3390/ app12020666

Academic Editor: Cristina Satriano

Received: 2 November 2021

Accepted: 5 January 2022

Published: 11 January 2022

Publisher's Note: MDPI stays neutral with regard to jurisdictional claims in published maps and institutional affiliations.

Copyright: (C) 2022 by the authors. Licensee MDPI, Basel, Switzerland. This article is an open access article distributed under the terms and conditions of the Creative Commons Attribution (CC BY) license (https:// creativecommons.org/licenses/by/ $4.0 /)$.
1 Department of Industrial Engineering, University of Florence, Via di Santa Marta 3, 50139 Florence, Italy; mattia.dimitri@unifi.it (M.D.); sara.aquino@unifi.it (S.A.); lucrezia.mazzantini@unifi.it (L.M.); martina.ricci2@stud.unifi (M.R.); andrea.corvi@unifi.it (A.C.)

2 Department of Experimental and Clinical Medicine, University of Florence, Viale Morgagni 50, 50134 Florence, Italy; claudia.duranti@unifi.it (C.D.); jessica.iorio@unifi.it (J.I.)

3 Department of Experimental and Clinical Biomedical Sciences "Mario Serio", University of Florence, Viale Morgagni 50, 50134 Florence, Italy; matteo.lulli@unifi.it

4 Department of Information Engineering, University of Florence, Via di Santa Marta 3, 50139 Florence, Italy; lorenzo.capineri@unfi.it

* Correspondence: annarosa.arcangeli@unifi.it; Tel.: +39-055-2751285; Fax: +39-055-2751281

+ Equally contributed.

$\ddagger$ Joint last authors.

\begin{abstract}
Current developments in medical technology have focused on therapeutic treatments that selectively and effectively address specific pathological areas, minimizing side effects on healthy tissues. In this regard, many procedures have been developed to provide non-invasive therapy, for example therapeutic ultrasound (US). In the medical field, in particular in cancer research, it has been observed how ultrasounds can cause cell death and inhibit cell proliferation of cancer cells, while preserving healthy ones with almost negligible side effects. Various studies have shown that low intensity pulse ultrasound (LIPUS) and low intensity continuous ultrasound (LICUS) regulate the proliferation, cell differentiation and cavitation phenomena. Nowadays, there are poorly known aspects of low intensity US treatment, in terms of biophysical and biomechanical effects on target cells. The aim of this study is to set up an innovative apparatus for US treatment of pancreatic ductal adenocarcinoma (PDAC) cells, monitoring parameters such as acoustic intensity, acoustic pressure, stimulation frequency and treatment protocol. To this purpose, we have developed a custom-made set up for the US stimulation at 1.2 and $3 \mathrm{MHz}$ of tridimensional (3D) cultures of PDAC cells (PANC-1, Mia Paca-2 and BxPc3 cells). Images of the 3D cultures were acquired, and the Calcein/PI assay was applied to detect US-induced cell death. Overall, the setup we have presented paves the way to an innovative protocol for tumor treatment. The system can be used either alone or in combination with small molecules or recombinant antibodies in order to propose a novel combined therapeutic approach.
\end{abstract}

Keywords: biological tissue; cancer therapy; LICUS; LIPUS; living cells; spheroids; ultrasound stimulation; controlled power wideband amplifier

\section{Introduction}

Besides systemic chemotherapy, local treatments, such as radiation and hyperthermia treatments, are typically used for cancer treatment. In these methods, the level of cell death induction is used as an indicator of therapeutic effectiveness. Despite a wide range of possible methodological options, there is a lack of valid strategies, based not only on the effect of high temperatures, but with a valuable effect per se. Among these, ultrasound technology has emerged as a possible novel therapeutic approach, despite the need for proper validation. 
Shock waves are heterogeneous, discontinuous, rapid mechanical phenomena that have been observed in the laboratory and in nature, in microscopic as well as in macroscopic dimensions, and in all states of matter.

In the management of solid cancer, the possible application of shock waves is ascribable to the pure mechanical effects on the cells. Differently, in addition to traditional surgery and chemotherapy, the use of high intensity focused ultrasound (HIFU) has been established as a noninvasive technique for tumor therapy.

In particular, the method of laser-induced shockwave generation is able to permeabilize cell membrane and increase drug delivery. Laser-induced methods for shock wave generation are reproducible on the macroscopic level and they have proven to prevent lithotripter related side-effects, such as heating and cavitation [1].

The research on ultrasound (US) therapy is focused on interactions of ultrasound mechanical energy delivered to biological tissues and the related phenomena that cause temperature rises. In the field of diagnostics and therapy, in fact, several simplified models have been developed to estimate the variation in temperature, inertial and non-inertial cavitation [2], and their biological consequences. The use of US for cancer treatment relies on both thermal and non-thermal effects, i.e., hyperthermia and acoustic streaming/radiation force, respectively.

US used in medicine can be divided into two main categories: US high intensity HIFU (high intensity focused ultrasound) and US low intensity LIU (low intensity ultrasound). The literature shows there is a limited value of the intensity from which the two types of ultrasounds are distinguished (Low: $0.5 \mathrm{MHz} \leq \mathrm{f} \leq 5 \mathrm{MHz}, 0.1 \mathrm{MPa} \leq \mathrm{P}_{\mathrm{a}} \leq 1.5 \mathrm{MPa}$; High: $\left.0.8 \mathrm{MHz} \leq \mathrm{f} \leq 7 \mathrm{MHz}, \mathrm{P}_{\mathrm{a}}>10 \mathrm{MPa}\right)[3]$.

Another classification of US is based on the intensity, ranging from $0.05-0.5 \mathrm{~W} / \mathrm{cm}^{2}$ for diagnostic imaging, to surgical applications with a range of $0.2-10 \times 10^{3} \mathrm{~W} / \mathrm{cm}$, to therapeutic applications that can use both high and low intensities [4]. An essential feature that characterizes ultrasounds is that, with increasing frequency, the attenuation for dissipative effects is very pronounced and it is therefore necessary to determine the value of the energy lost in propagation.

Among different US, high intensity focused ultrasound (HIFU) has been investigated extensively and found to be highly effective in treating different cancers via hyperthermia. The US has also been very effective in creating stroma in the cell membrane thus enhancing the drug intake. Tumor cells demonstrated the ability to acquire thermo-resistance due to repeated hyperthermia and the efficiency of hyperthermia-based treatments, hence, decreases with repeated use. Therefore, among magnetic and US based treatments, the latter demonstrated more effectiveness and strong application potential.

Low intensity pulsed ultrasound (LIPUS) is a technology that can be used for therapeutic purposes. It exploits low intensity and pulsed mechanical waves to induce regenerative and anti-inflammatory effects on biological tissues, such as bone, cartilage, and tendons. Even if the real mechanism underlying its effectiveness has not been understood yet, it is plausible that the treatment relies on non-thermal phenomena, such as microbubbles and microjets induced by cavitation, acoustic streaming, and mechanical stimulation. The typical frequency range of LIPUS is between $40 \mathrm{kHz}$ and $5 \mathrm{MHz}$ with intensity levels between 5 and $1000 \mathrm{~mW} / \mathrm{cm}^{2}$ and the commonly burst excitation used is characterized by a pulse width of $200 \mu$ s and pulse repetition frequency (PRF) of $1 \mathrm{kHz}$, corresponding to a duty cycle of $20 \%$. LIPUS is used for stimulating healing in different tissues for a variety of applications, especially in rehabilitation medicine, bone-fracture healing, soft-tissue regeneration, and inflammatory responses inhibition. At a cellular level, LIPUS has also been observed to increase migration and proliferation of aortic endothelial cells, proliferation of fibroblasts, Schwann cells, and other cell types [5].

While the effect of HIFU is associated with generated heat, the therapeutic effects of LIPUS are traced back to low intensities within the range of $0.07-3 \mathrm{~W} / \mathrm{cm}^{2}$ Spatial Average Temporal Average (SATA) [6] and frequencies of $0.5-5 \mathrm{MHz}$ and a treatment duration of 5-20 min per day [7]. Many studies showed evidence that LIPUS could regulate cell 
proliferation and differentiation [8]. Induced biomechanical effects, such as radiation force, acoustic streaming, and strain gradients, seem to play a major role in triggering cellular responses at LIPUS exposures.

In order to provide good understanding on the effects of magnetic fields and US on cancer cells and tissues, further studies are required to establish their efficiency in clinical environments. For example, comparison of results in different literature appears to be very difficult due to large variations in US treatment conditions [9].

Pancreatic Ductal Adeno Carcinoma (PDAC) is one of the most common causes of cancer-related death with a 1-year survival rate lower than $20 \%$ and a 5 -year survival rate of $8 \%$, regardless of the stage. The disease is almost always fatal, and its incidence is forecasted to continuously grow in the next years, unlike other cancer types, whose incidence is declining. For these reasons, PDAC represents an urgent medical need, and the accomplishment of new treatments is therefore mandatory. The aim of this study is the research and optimization of the parameters for the ultrasound treatment of pancreatic cancer cell lines, PANC-1, Mia PaCa-2 and BxPc3, in order to induce a resonance effect in cell capable of damaging them and eventually leading to cell death [10].

\section{Materials and Methods}

\subsection{Experimental Set-Up}

The ultrasonic setup (Figure 1) for cell stimulation is composed of a programmable sinusoidal wave generator (MS-9160 by Universal System, Hirschau, Germany) connected in series to a power amplifier and an immersion US transducer (WS75-2, Ultran Group) with an active diameter of $19 \mathrm{~mm}$. The controller can read the amplified signal by monitoring the input signal to ensure the proper stimulation signal power. A broadband linear power amplifier designed by the University of Florence was used [11]. An oscilloscope, HANTECK DSO4102C (Qingdao Hanteck Electronics \& Co. Qingdao, Shandong, China), is connected to the linear power amplifier to measure the actual signal amplitude applied to the probe and the relative power when excited in continuous wave (CW) mode. The low distortion of this amplifier [10], even at very low transducer impedance, makes it suitable to define the ultrasound power parameters. The transducer and the sample were coaxially aligned in a small tank of deionized and degassed water of dimension $400 \times 150 \times 100 \mathrm{~mm}$ and $5 \mathrm{~mm}$ thickness. To allow the probe to move in the three directions, it was placed on a mechanical support that allows the US beam to be centered on the target spheroids using a laser pointer.

All cell stimulation experiments were performed in water with samples positioned at the point of maximum pressure. The control of the latter is an important task for understanding the biomechanical and biophysical effects and a detail description will be provided in this section.

Nowadays, in vitro and in vivo studies are conducted on the biological effects of low intensity ultrasound stimulations to find out the basic mechanisms and parameters to focus on in order to optimize the treatment of cancer cells.

The experimental setup involved the Ultran WS75-2 (Ultran Group, State College, PA 16801 USA) ultrasound probe, made of lead metaniobate piezoelectric material (PbNb2O6) and characterized by a geometric focus, a nominal frequency of $2 \mathrm{MHz}$ and a frequency band between $1 \mathrm{MHz}$ and $3.5 \mathrm{MHz}$ (Ultran Group, State College, PA $16801 \mathrm{USA}$ ).

In particular, water was used as the transmission medium for its extremely small attenuation coefficient, equal to $0.002 \mathrm{dBcm}^{-1} \mathrm{MHz}^{-1}$ at $25^{\circ} \mathrm{C}$, resulting in an acceptable attenuation for the probe focal distance, calculated at the three respective stimulation frequencies.

For the stimulation at the three different frequencies, which correspond the three different focal distances, a slide was made on the bottom of the tank allowing the probe to be fixed at the required focal distance. The probe support is designed to allow translation on all three axes, keeping the US beam perpendicular to the surface of the multiwell containing cell cultures (Figure 2). 


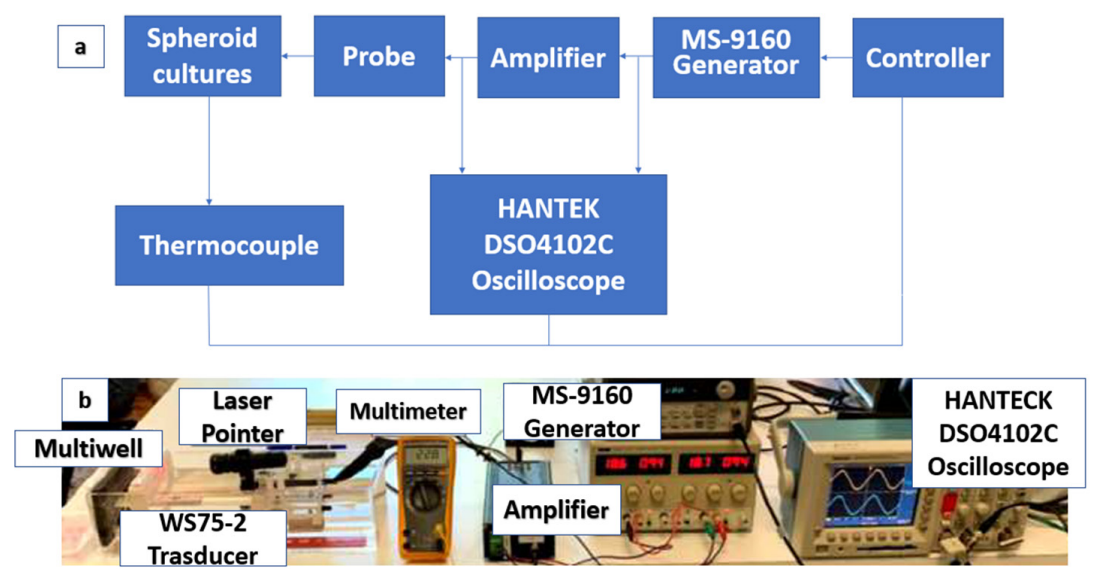

Figure 1. (a) Block scheme with connections of the instruments used for the experimental set up. The spheroid cultures are stimulated by the ultrasonic probe (WS75-2 Transducer) which is connected to a generator of sinusoidal functions (MS-9160 Generator). Between the probe and the generator is located the amplifier, designed for the purpose of amplifying the signal in this specific application. The oscilloscope is connected before and after the amplifier to verify that the signal is amplified correctly. The controller block indicates a carry out on the output voltage from the generator to protect the instrumentation from malfunctions. The thermocouple has the function of controlling the rise in temperature, both to check that it does not rise excessively, inducing death by hyperthermia in the spheroid's cultures, and to check the presence of ultrasound. (b) In the picture it is shown how the experimental set up is presented during the experimentation. The spheroid cultures are located in the multiwell and the presence of the laser pointer is aimed at centering with the probe the spheroid designated to be stimulated. The plexiglass structure that supports the probe provides a guide to move it at different focal distances. The multimeter acts as a thermocouple.

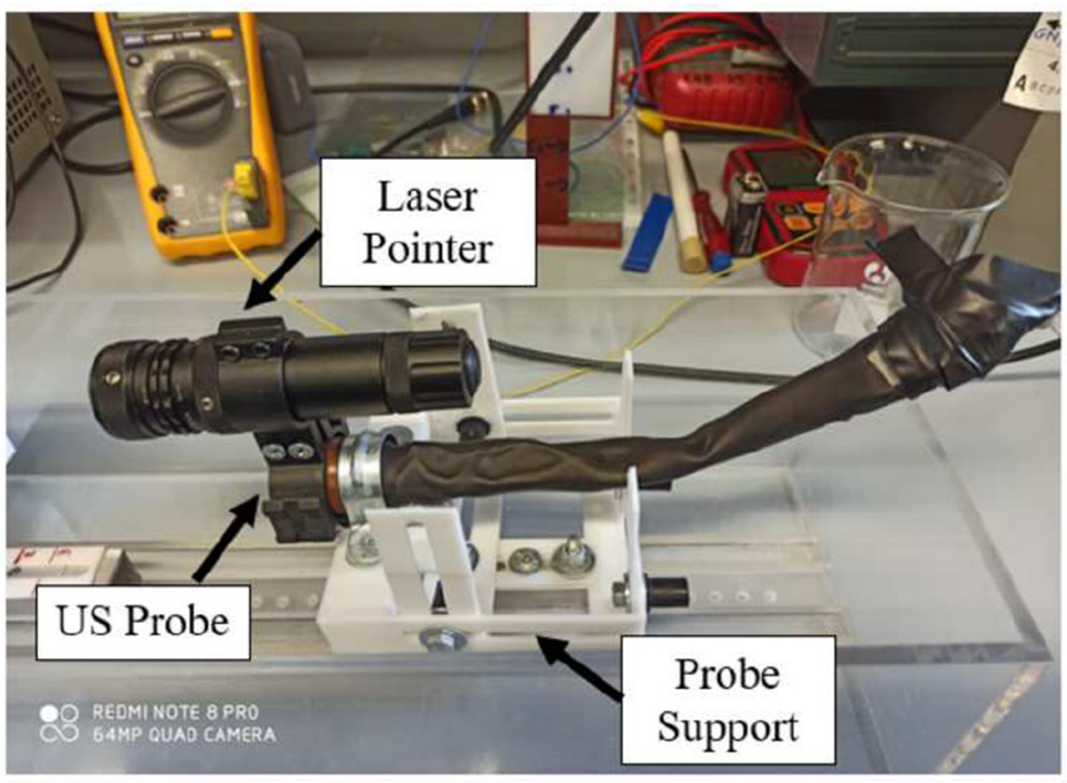

Figure 2. The picture shows the detail of the guide on which the probe can be moved, attached to the laser pointer, to move at different focal distances according to the frequency.

\subsection{Cell Culture and Methodology Description}

The heating inside the multiwell was monitored and quantified using a mixture consisting of agarose and culture medium was prepared: the thermocouple was positioned inside the mixture, the multiwell was sealed, and stimulation was started.

For experimental tests, PANC-1 (ATCC-LGC, Manassas, VA, USA), human pancreatic ductal adenocarcinoma cell line along with Mia Paca2 (ATCC-LGC, Manassas, VA, USA) 
and BxPc3 (ATCC-LGC, Manassas, VA, USA) human pancreatic cancer cell lines were stimulated. All the cells were purchased from ATCC (American Type Culture Collection).

Thanks to the probe field intensity characterization it was possible to establish for each working frequency where to place cells cultures within a 96-well multiwell (Fisher Scientific Italia, Rodano (MI) Italy) avoiding the overlap of the US beam. This configuration has permitted the testing of major number of spheroids in the same day test, reducing the cell mortality by quiescent effect. Each multiwell has four spheroids to stimulate and four control spheroids, as shown in Figure 3. Since four multiwells (one for each stimulation frequency) have been prepared for the experimental tests, the results obtained from each frequency have been evaluated on four different cultures. It was important to insert the control group inside the multiwell, because they had to stay in the same environmental and suffer transportation, immersion in water at $20^{\circ} \mathrm{C}$, to a good comparison.

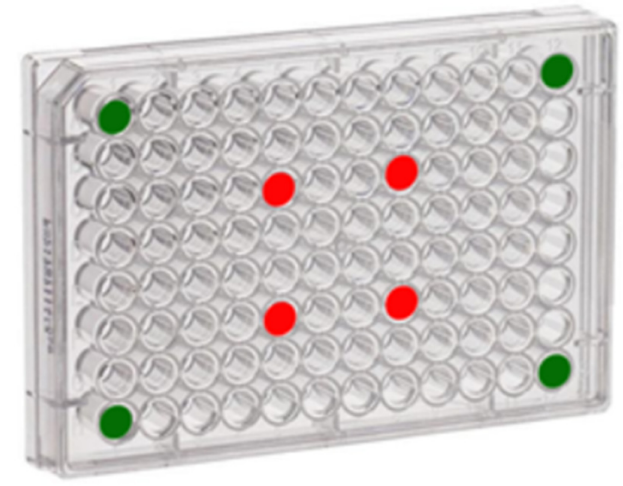

Figure 3. The figure shows the positioning of the control spheroids (green) and of those that will be treated (red). The probe beam is $16 \mathrm{~mm}$ in diameter so two wells are sufficient to avoid overlapping of effects.

To avoid water infiltration inside the multiwell, the latter was sealed using a transparent PVC film positioned between the multiwell and the multiwell cover. By properly adhering the film to the multiwell and sealing the system, it is possible to avoid contamination of cell cultures.

For every test, PANC-1 cells were cultured in an up-to-date 3D conformation, which is that of a spheroid. The latter are usually non-scaffold-based cultures formed by rounded cellular aggregates [12]. Cells were routinely cultured in a humidified incubator at $37^{\circ} \mathrm{C}$ with $5 \% \mathrm{CO}_{2}$ using DMEM Dulbecco's Modified Eagle Medium (DMEM) (Fisher Scientific Italia, Rodano (MI), Italy), High Glucose (Euroclone S.p.A, Pero (MI), Italy) supplemented with 4 mM L-Gln (Glutamine) and 10\% FBS (Fetal Bovine Serum) (Euroclone S.p.A, Pero (MI), Italy). For 3D spheroid cultures, cells were detached with trypsin and counted using trypan blue. Trypan blue dye exclusion test was used to determine the number of viable cells present in a cell suspension. It is based on the principle that live cells possess intact cell membranes that exclude certain dyes, such as trypan blue. The $10^{3}$ cells were seeded on an agarose base layer $(1.5 \mathrm{~g} / \mathrm{l})$ in a 96-well plate and grown for $96 \mathrm{~h}$ in a humidified incubator at $37^{\circ} \mathrm{C}$ and $5 \% \mathrm{CO}_{2}$.

All four spheroids for each stimulation frequency were treated for $15 \mathrm{~min}$. Before proceeding, the initial temperatures of the water contained in the tank and the wells containing the target cultures were acquired. The same acquisitions were repeated every 5 min until the end of the treatment for a total of five acquisitions, in order to verify a thermal increase of less than $2{ }^{\circ} \mathrm{C}$ at the target well. The 96 -well plate after the incubation in the experimental set up was immediately moved to the cell incubator using a portable $37^{\circ} \mathrm{C}$ incubator set up with controlled temperature parameters.

\subsection{Calcein $A M$ and Annexin/PI Assay}

Calcein AM/ / PI (AcetoxyMethyl/Propidium Iodide) is a cell dye that can be used to determine cell viability in most eukaryotic cells. In live cells the nonfluorescent calcein 
AM is converted to a green, fluorescent calcein after acetoxymethyl ester hydrolysis by intracellular esterases. PI is internalized by necrotic cells. For Calcein AM/PI assay, cells were stained by adding fresh medium containing Calcein AM $(2 \mu \mathrm{g} / \mathrm{mL})$ and PI $(10 \mu \mathrm{g} / \mathrm{mL})$ (Euroclone S.p.A, Pero (MI), Italy), incubated for $20 \mathrm{~min}$ and then washed with PBS. The samples were examined using an SP8 confocal microscope (Leica, Wetzlar, Germany). Images were captured using the Leica LAS-X image acquisition software (Leica Microsystems GmbH, Wetzlar, Germany).

Apoptosis was determined using the Annexin V/PI test (Annexin-V FLUOS Staining Kit; Roche Diagnostics, Mannheim, Germany), as in [12]. Spheroids were taken from the agarose using a micropipette with an enlarged tip, in order to achieve high harvesting efficiency with great spheroid integrity. The harvested spheroids collected using manual pipetting at this point were dissociated into single cells using trypsin-EDTA $0.25 \%$ for $5 \mathrm{~min}$ with gentle pipetting up and down to minimize aggregated cell population for following analysis [13]. According to this procedure, (i) viable cells are negative for both Annexin V and PI (Q3 quadrant gate in the dot plots); (ii) cells that are in the early phases of apoptosis are Annexin V positive and PI negative (Q4 quadrant gate in the dot plots); (iii) cells that are in the late phases of apoptosis are both Annexin V and PI positive (Q2 quadrant gate in the dot plots); and (iv) dead cells are Annexin V negative and PI positive (Q1 quadrant gate in the dot plots).

\subsection{Statistical Analysis}

Unless otherwise indicated, data are given as mean values \pm SEM. At least three independent experiments were performed. Statistical comparisons were performed with OriginPro 2015 and SAS 9.2 (SAS Institute) software. The normality of data distribution was checked with a K-S test. In the case of normal distributions, each data set was first checked for variance homogeneity, using the $\mathrm{F}$ test for equality of two variances and the Brown-Forsythe test for multiple comparisons. For data with unequal variances, the Welch correction was applied. For comparisons between two groups of data, we used the Student's $t$ test. A two-sample K-S test was performed to test whether two underlying probability distributions differed. For multiple comparisons, one-way ANOVA followed by Bonferroni's post hoc test was performed to derive $p$ values.

\subsection{Stimulation Parameters}

In this set up we used a $15 \mathrm{MHz}$ bandwidth linear power amplifier [11] and a hydrophone (0.25-20 MHz frequency range, sensitivity: $282 \mathrm{nV} / \mathrm{Pa}$, HGL 0400 series by Onda Sonics Corp. Sunnyvale, CA, USA) was used to determine the trend of the probe pressure range and the US focus. Spheroids were treated at three different stimulation frequencies and then each well containing a treated spheroid was stained using Calcein AM/PI dye. Subsequently, spheroid images were acquired using a confocal fluorescence microscope, and cell vitality vs. mortality was assessed using the BioFilmAnalyzer software [14].

For $f=1 \mathrm{MHz}$ stimulation, a value of $\mathrm{F}=90 \mathrm{~mm}$ from the transducer was chosen as the focal distance since it is the first point on the interpolation curve (Figure 4). This point is located in the far field and with the chosen parameters the Rayleigh length $\left(Z_{R}\right)$ results at $55 \mathrm{~mm}$ from the Ultrasound probe surface. The Rayleigh length is an important parameter because it represents the distance along the propagation direction of the beam from the waist to the place where the area of the cross section is doubled, and it is commonly use when beams are modeled as Gaussian beams:

$$
z_{R}=\frac{a^{2}}{\lambda}=\frac{a^{2} f}{c}=55 \mathrm{~mm}
$$

where $a$ is the radius of the piezoelectric and $c$ is velocity of propagation in the medium.

For $2 \mathrm{MHz}$ stimulation (Figure 5), measurements were taken around the Rayleigh distance $(110 \mathrm{~mm})$. In fact, in the graph there are points that give an irregular trend and that 
come out of the interpolation curve $(80,90$ and $100 \mathrm{~mm})$. The point chosen for stimulation is at a distance of $120 \mathrm{~mm}$ from the translator.

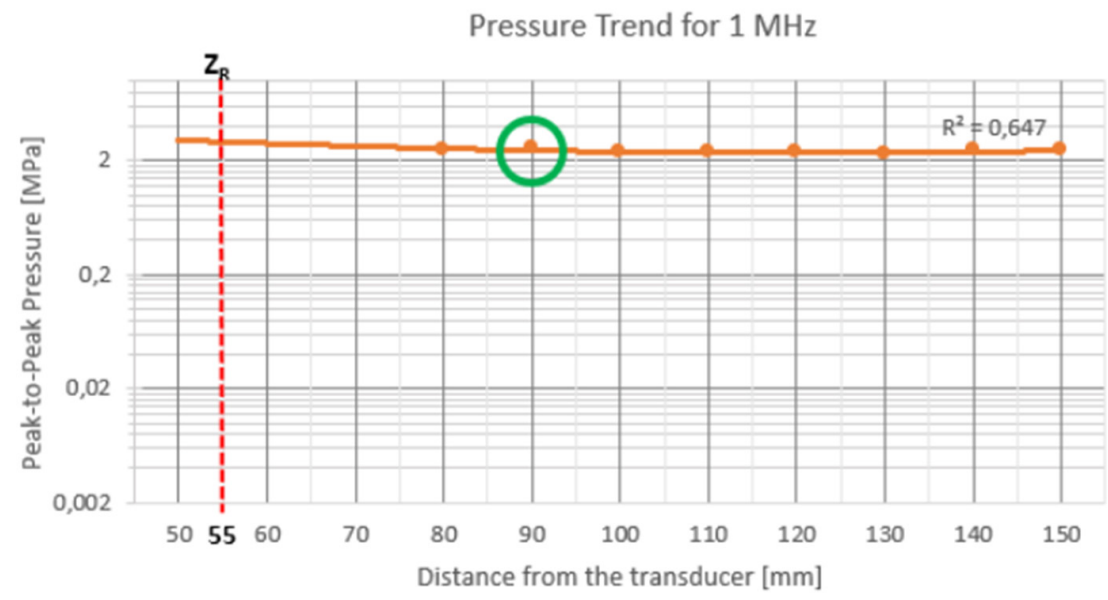

USCND Lab trend

Figure 4. Focal distance calculation. At $1 \mathrm{MHz}$ stimulation frequency the selected stimulation point is at a distance of $90 \mathrm{~mm}$ from the transducer (green circumference), this point in fact results in the Far Field. This value results from a test made with the hydrophone and by a subsequent linear interpolation. $Z_{R}$ indicates the distance of Rayleigh (dotted line in red). The orange line in the figure indicates the peak-to-peak pressure trend carried out by the Ultrasound and Non Destructive Testing (USCND) laboratory of the Dept. of Information Engineering of the University of Florence.

\section{Pressure trend for $2 \mathrm{MHz}$}

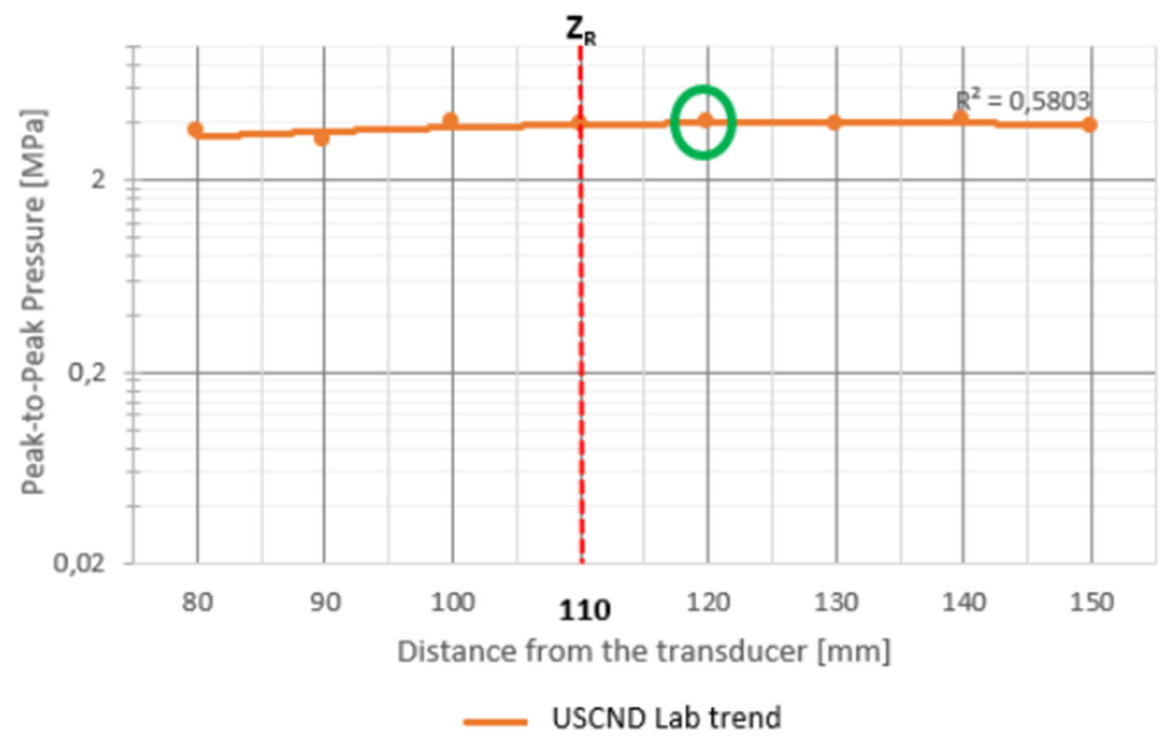

Figure 5. Focal distance calculation. At $2 \mathrm{MHz}$ stimulation frequency the selected stimulation point is at a distance of $120 \mathrm{~mm}$ from the transducer (green circumference). This value results from the test made with the hydrophone and by a subsequent linear interpolation. $Z_{R}$ indicates the distance of Rayleigh (dotted line in red), in this case the $Z_{R}$ is located right after the cut-off region where the pression is very uneven. The orange line in the figure indicates the peak-to-peak pressure trend made by the USCND laboratories.

For $3 \mathrm{MHz}$ stimulation (Figure 6), the points measured by the hydrophone are located in the near field, in fact $Z_{R}=165 \mathrm{~mm}$. The only point that is positioned around the Rayleigh distance is $150 \mathrm{~mm}$, chosen as the optimal stimulation point. 


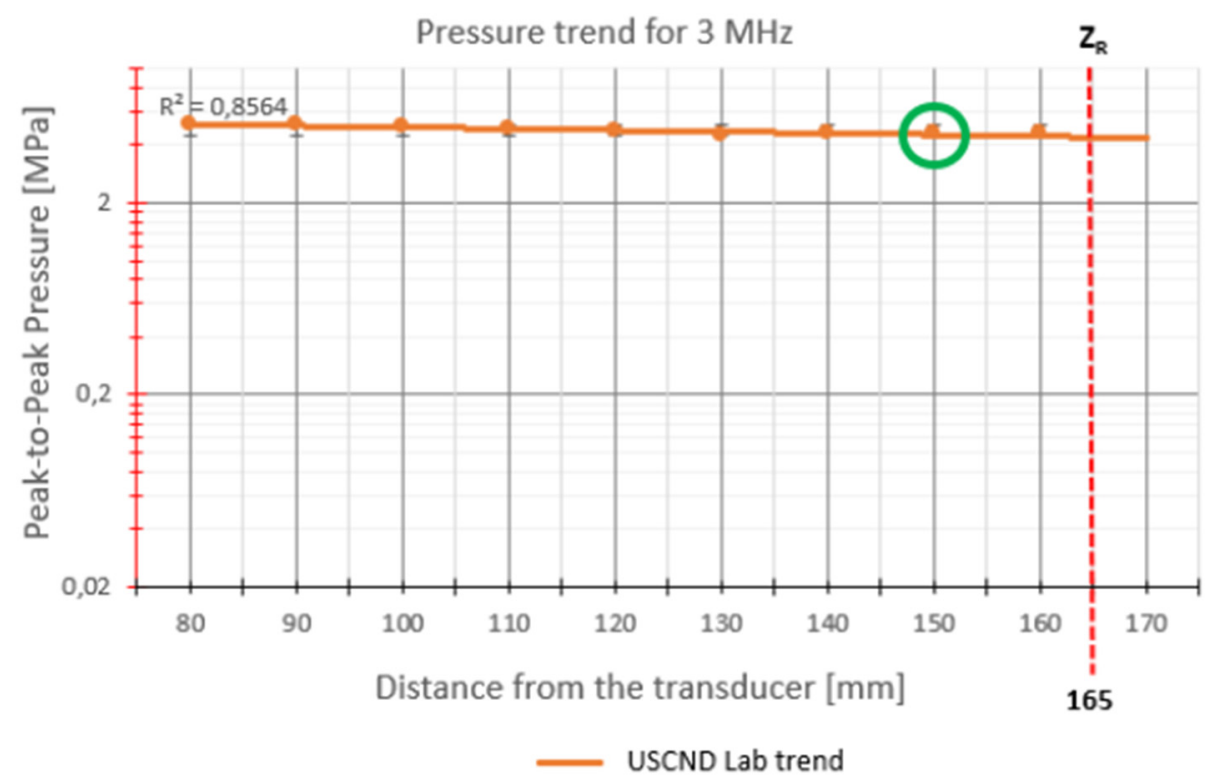

Figure 6. Focal distance calculation. At $3 \mathrm{MHz}$ stimulation frequency the selected stimulation point is at a distance of $150 \mathrm{~mm}$ from the transducer (green circumference). This value results from the test made with the hydrophone and by a subsequent linear interpolation. $Z_{R}$ indicates the distance of Rayleigh (dotted line in red), in this case the $Z_{R}$ is located right after the cut-off region where the pression is very uneven. The orange line in the figure indicates the peak-to-peak pressure trend made by the USCND laboratories.

To calculate the intensity, the following aspects were considered:

- The difference between the acoustic impedances of the water and the multiwell cover generate a reflection of the pressure wave, which can be assessed with the following formula [11]:

$$
p_{i} p_{t}=T=1-Z_{p}-Z_{a} Z_{p}+Z_{a}=0.62
$$

where $p_{i}$ and $p_{t}$ are the pressure incident on the cover of the multiwell and the pressure transmitted through the cover, respectively. $T$ is the transmission coefficient. $Z_{p}$ and $Z_{a}$ are respectively the acoustic impedance of the PMMA cover (3.23 MRayls) and of the water (1.48 MRayls).

- The attenuation of the sound beam through the cover will be minimal and can be assessed using the following formula [11]:

$$
p(x)=p(0) e^{-\alpha \cdot x}
$$

where $\alpha=1.1 \mathrm{~dB} /(\mathrm{cm} \mathrm{MHz})$ is the attenuation coefficient of PMMA, $x$ is the distance traveled by the beam through the material, $p(0)$ the initial pressure.

According to the previous considerations, the SATA values were calculated considering the Spatial Peak Intensity ( $\left.\mathrm{I}_{\mathrm{SP}}\right)$, that represents the energy provided by the beam on the area of $1 \mathrm{~cm}^{2}$, at the three stimulation frequencies $\left(\mathrm{I}_{\mathrm{sp} 1}=1 \mathrm{Mhz} ; \mathrm{I}_{\mathrm{sp} 2}=2 \mathrm{Mhz} ; \mathrm{I}_{\mathrm{sp} 3}=3 \mathrm{Mhz}\right)$, considering a Gaussian waveform of the beam (whence the Beam Uniformity Ratio) and a duty cycle of $20 \%$, obtaining the following values:

$$
\begin{gathered}
I_{S P 1}=\frac{p^{2}}{2 \cdot Z}=17.514 \frac{\mathrm{W}}{\mathrm{cm}^{2}} \\
I_{S P 2}=34.133 \frac{\mathrm{W}}{\mathrm{cm}^{2}}
\end{gathered}
$$




$$
\begin{gathered}
I_{S P 3}=36.471 \frac{\mathrm{W}}{\mathrm{cm}^{2}} \\
S A T A_{1}=1.167 \frac{\mathrm{W}}{\mathrm{cm}^{2}} \\
S A T A_{2}=2.275 \frac{\mathrm{W}}{\mathrm{cm}^{2}} \\
S A T A_{3}=2.431 \frac{\mathrm{W}}{\mathrm{cm}^{2}}
\end{gathered}
$$

These SATA values fall within the characteristic range of the low intensities $0.07-3 \mathrm{~W} / \mathrm{cm}^{2}$ of ultrasound that involves the temporal average of all propagating pulses that are emitted during several frames and that make significant intensity contributions over a specified scan cross-sectional area.

\section{Results}

The thermal trends recorded during the stimulation procedure confirm a low intensity treatment; in fact, cell death is not caused by thermal effects, but only by the mechanical effects of ultrasound. The temperatures were acquired in water, in correspondence with the probe and inside the target well. Tables 1-3 show the average values calculated from the four stimulations conducted for each frequency.

Table 1. Average temperature values calculated at $1 \mathrm{MHz}$.

\begin{tabular}{cccc}
\hline \multicolumn{4}{c}{ Spheroids at $\mathbf{1} \mathbf{~ M H z}$} \\
\hline Time (min) & Twater $\left({ }^{\circ} \mathbf{C}\right)$ & Tpiezo $\left({ }^{\circ} \mathbf{C}\right)$ & Ttarget Well $\left({ }^{\circ} \mathbf{C}\right)$ \\
\hline 0 & 18.9 & 19.1 & 19.0 \\
5 & 19.0 & 19.4 & 19.2 \\
10 & 19.1 & 19.5 & 19.2 \\
15 & 19.1 & 19.5 & 19.2 \\
20 & 19.0 & 19.5 & 19.2 \\
\hline
\end{tabular}

Table 2. Average temperature values calculated at $2 \mathrm{MHz}$.

\begin{tabular}{cccc}
\hline \multicolumn{4}{c}{ Spheroids at $\mathbf{2} \mathbf{~ M H z}$} \\
\hline Time (min) & Twater $\left({ }^{\circ} \mathbf{C}\right)$ & Tpiezo $\left({ }^{\circ} \mathbf{C}\right)$ & Ttarget Well $\left({ }^{\circ} \mathbf{C}\right)$ \\
\hline 0 & 19.5 & 19.8 & 19.7 \\
5 & 19.6 & 21.1 & 19.8 \\
10 & 19.6 & 21.1 & 19.8 \\
15 & 9.6 & 21.0 & 19.8 \\
20 & 19.7 & 21.1 & 19.8 \\
\hline
\end{tabular}

Table 3. Average temperature values calculated at $3 \mathrm{MHz}$.

\begin{tabular}{cccc}
\hline \multicolumn{4}{c}{ Spheroids at $\mathbf{3 ~ M H z}$} \\
\hline Time (min) & Twater $\left({ }^{\circ} \mathbf{C}\right)$ & Tpiezo $\left({ }^{\circ} \mathbf{C}\right)$ & Ttarget Well $\left({ }^{\circ} \mathbf{C}\right)$ \\
\hline 0 & 20.4 & 20.8 & 20.6 \\
5 & 20.5 & 22.3 & 20.8 \\
10 & 20.4 & 22.2 & 20.8 \\
15 & 20.4 & 22.4 & 20.8 \\
20 & 20.5 & 22.4 & 20.8 \\
\hline
\end{tabular}

Ten sessions of experimental testing took place. For each treatment, three 96-well plates were used, one for each stimulation frequency, including four containing PANC-1 cell cultures to be treated, and four containing control PANC-1 cell cultures. 
Cells treated at different frequencies were stained with Calcein AM/PI and images were acquired using Confocal microscopy at a magnification of $10 \times$ (image size for all pictures: $900 \times 900 \mu \mathrm{m}$ ). The analysis provided cell death index as a ratio between red cells (dead cells) and green cells (live cells), as shown in Figure 7. The percentage of dead cells was shown at three different frequencies for the treated spheroids. On the right side of each panel, the mortality rate of untreated spheroids due to the possible side effects induced at various frequencies at a distance of $25 \mathrm{~mm}$ from the stimulation beam was evaluated.

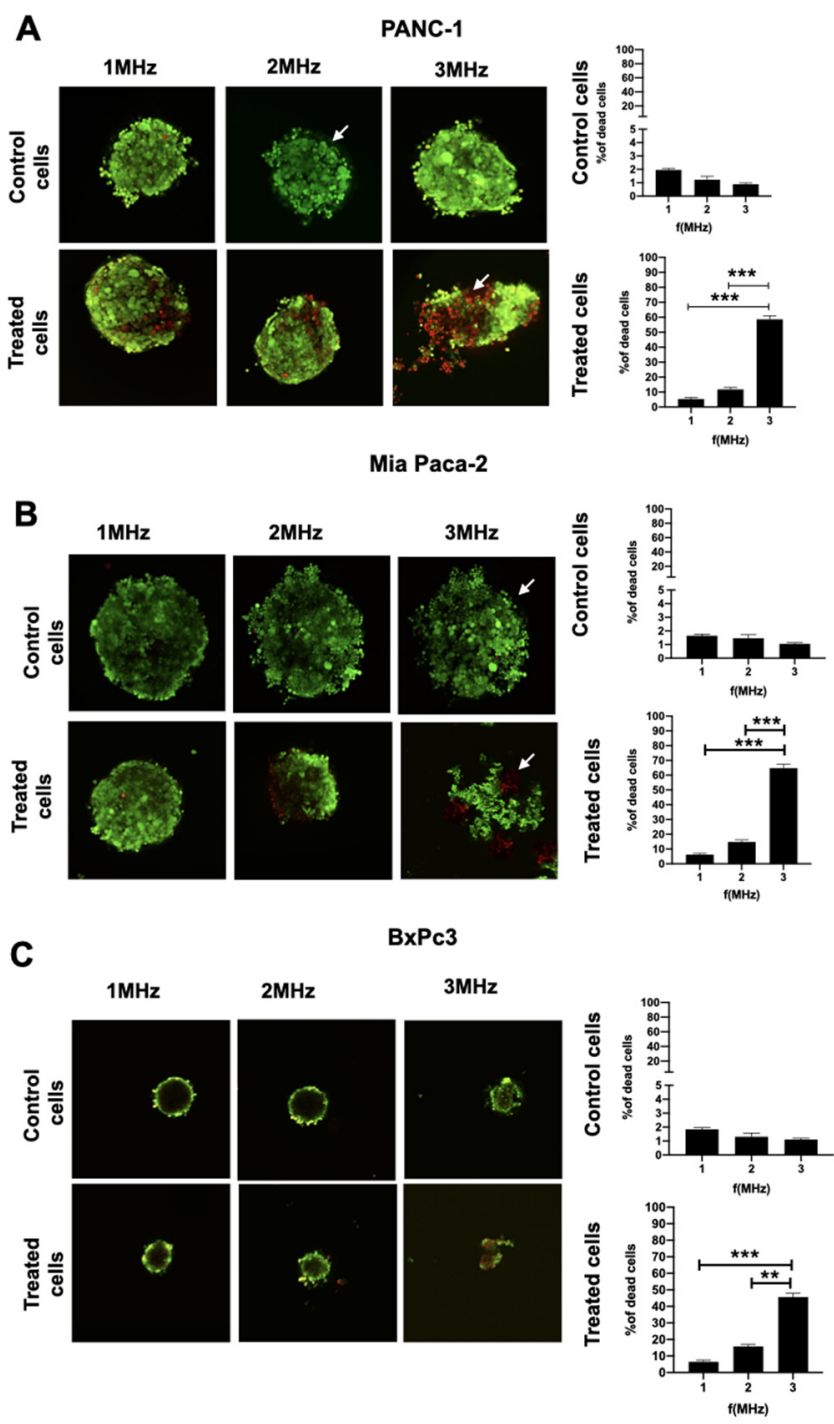

Figure 7. Images acquired with Confocal fluorescence microscope SP8 confocal microscope (Leica), showing PDAC cell spheroids. (A) PANC-1 spheroids, control upper panel and treated lower panel with 1, 2 and $3 \mathrm{MHz}$. In each well one spheroid was seeded, and images were acquired at $10 \times$ magnification. Control cells appear stained in green, which represents viable cells (see white arrows); while treated cells show a marked spotted red staining (see white arrow), which represent dead cells. Quantification of viable and dead cells using the BioFilmAnalyzer software is also reported. (B) Mia Paca-2 spheroids, control cells upper panel and treated cells lower panel, with 1, 2 and $3 \mathrm{MHz}$. In each well one spheroid was seeded, and images were acquired at $10 \times$ magnification. Control cells appear with a predominant green staining (see white arrow), indicating viable cells; while treated spheroids appear smaller and with a prevalence of red staining dots, indicating cell death (see white arrows). Mia Paca-2 spheroids, treated with $3 \mathrm{MHz}$ frequency, also appear to disaggregate. Quantification of viable and dead cells using Spheroid Sizer (Mathlab) is also reported. (C) BxPc3 spheroids, control upper panel and treated lower panel with 1, 2 and $3 \mathrm{MHz}$. In each well one spheroid was seeded, and 
images were acquired at $10 \times$ magnification. Control cells appear stained in green, which represents viable cells (see white arrows); while treated cells show a marked spotted red staining. BxPc3 spheroids appear smaller, as per proper cell characteristics. Cell staining appears concentrated on the spheroid margins, as they are compact and have less dye permeable. Quantification of viable and dead cells using Spheroid Sizer (Mathlab) is also reported. Image size for all pictures: $900 \times 900 \mu \mathrm{m}$, acquired under $10 \times$ magnification. ${ }^{* * *} p<0.001{ }^{* *} p<0.01$.

$p$-Values for each investigated frequency results were: 0.19 for $1 \mathrm{MHz}, 0.0018$ for $2 \mathrm{MHz}$ and 0.0013 for $3 \mathrm{MHz}$, respectively. Highly significant values were obtained using $3 \mathrm{MHz}$ frequencies, if compared to both 1 and $2 \mathrm{MHz}$.

During the US treatment, total disruption of some spheroids was found with a cadency of $25 \%$ within the treated population. The frequency seems to affect only the level of cell scattering, as shown in Figure 8. Overall, the morphology of the spheroids results appeared to be deeply affected by the US treatment. Spheroids appeared more compact in the controls, while cells tended to disaggregate after the treatment, losing their shape or appearing enlarged and round-shaped at the highest frequency of $3 \mathrm{MHz}$. After treatment, $\mathrm{BxPc} 3$ cells tended to disrupt and to move from the center core of the spheroids.

A
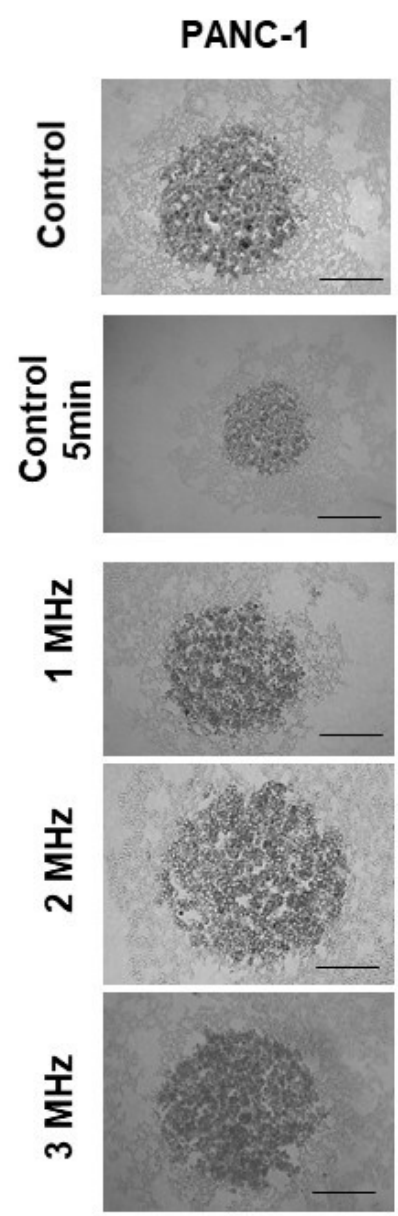

B

\section{Mia Paca-2}
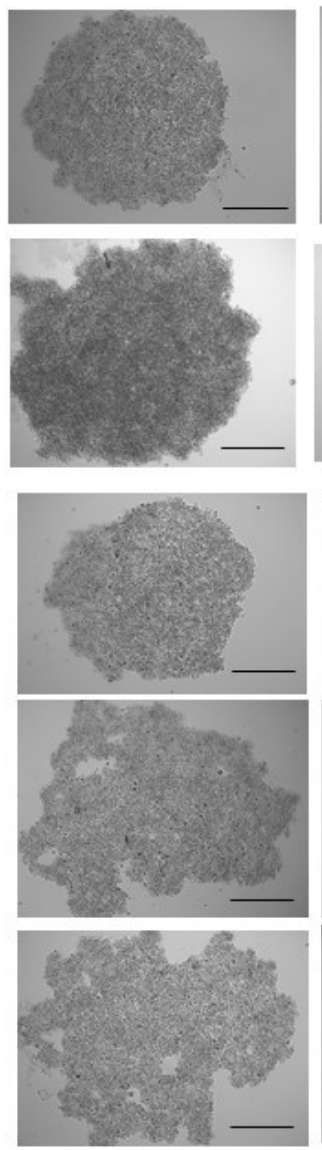

C

BxPc3
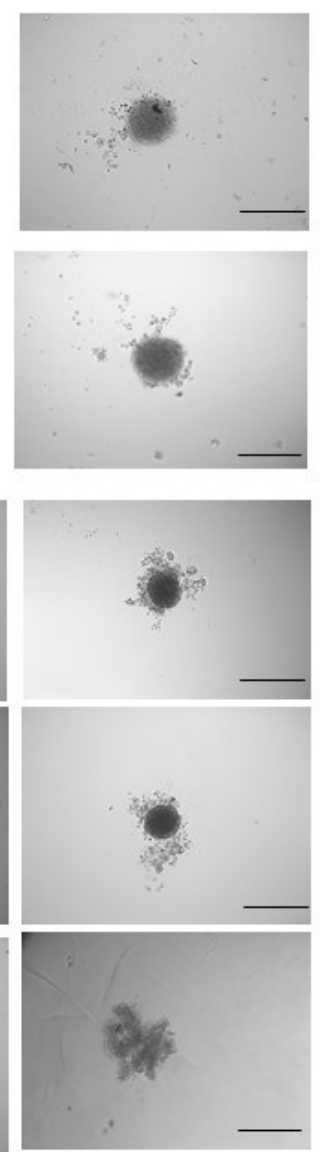

Figure 8. Brightfield images of PANC-1, Mia Paca 2 and BxPc3. (A) PANC-1 images of spheroids, control (untreated cells), control after $5 \mathrm{~min}$ (Control $5 \mathrm{~min}$ ) of stimulation, cells treated with $1 \mathrm{MHz}$, $2 \mathrm{MHz}$ and $3 \mathrm{MHz}$. (B) Mia Paca 2 images of spheroids, control (untreated cells), control after $5 \mathrm{~min}$ (Control $5 \mathrm{~min}$ ) of stimulation, cells treated with $1 \mathrm{MHz}, 2 \mathrm{MHz}$ and $3 \mathrm{MHz}$. (C) BxPc3 images of spheroids, control (untreated cells), control after $5 \mathrm{~min}$ (Control $5 \mathrm{~min}$ ) of stimulation, cells treated with $1 \mathrm{MHz}, 2 \mathrm{MHz}$ and $3 \mathrm{MHz}$ (scale bar: $100 \mu \mathrm{m}$ ). 
We also analyzed the Annexin/PI staining in all the three cell lines, comparing the results in control conditions, as well as after the application of $3 \mathrm{~Hz}$ US. Figure 9 clearly shows that all the three PDAC cells are in late apoptosis after US treatment at $3 \mathrm{MHz}$, compared to the respective control.

Control cells

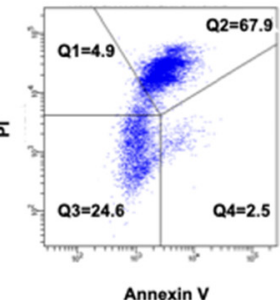

Control cells
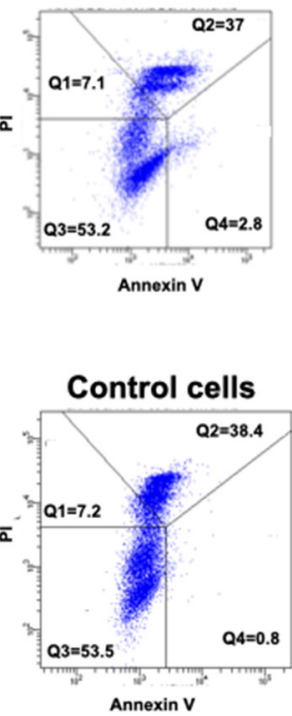

$\bar{a}$

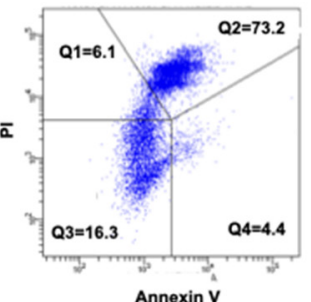

PANC-1
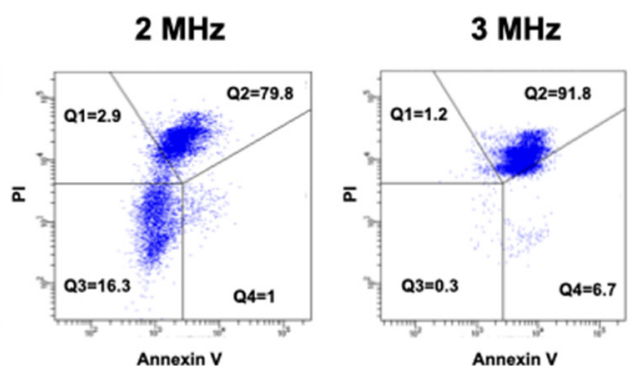

\section{Mia Paca-2}

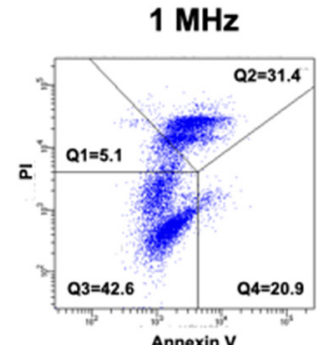

$2 \mathrm{MHz}$

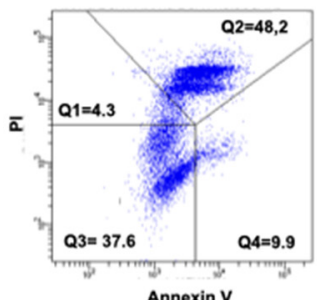

BxPc3
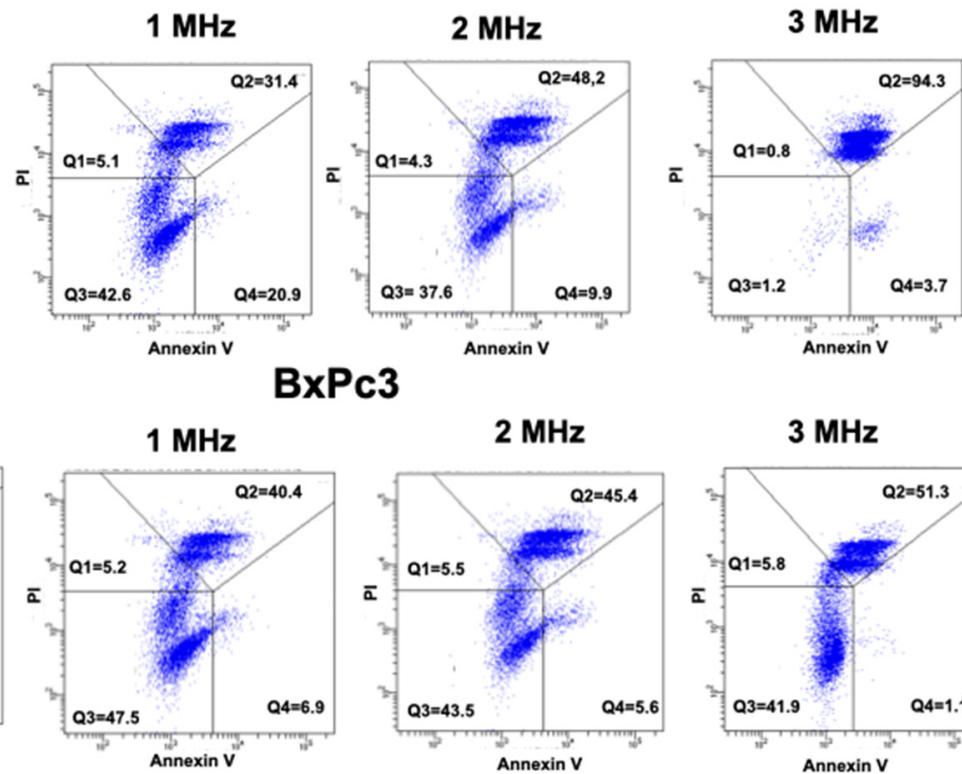

$3 \mathrm{MHz}$

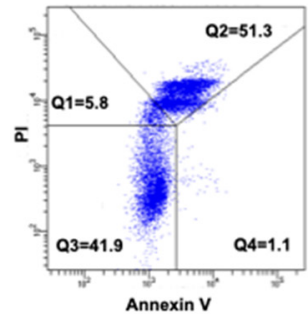

Figure 9. Results of Annexin Pi staining performed on PANC-1, Mia Paca 2 and BxPc3 cells. Viable cells are negative for both Annexin V and PI (Q3 quadrant gate in the dot plots); cells that are in the early phases of apoptosis are Annexin V positive and PI negative (Q4 quadrant gate in the dot plots); cells that are in the late phases of apoptosis are both Annexin V and PI positive (Q2 quadrant gate in the dot plots); and dead (necrotic) cells are Annexin V negative and PI positive (Q1 quadrant gate in the dot plots).

In particular, PANC-1 cells show 91.8\% of treated cells in Q2 (late apoptosis) compared to $67.9 \%$ of Control cells. Mia Paca-2 treated cells have $94.3 \%$ of cells in Q2, compared to $37 \%$ of Control cells. Finally, BxPc3 has 51.3\% of cells in Q2 compared to $38.4 \%$ of control cells. A lower effect, but in consistence with the trend, was envisaged in cells treated at lower frequencies, as reported in Figure 9.

\section{Discussion}

Literature describes the use of LIPUS in four major areas of cancer therapy: sonodynamic therapy, ultrasound-mediated chemotherapy, ultrasound-mediated gene delivery and anti-vascular ultrasound therapy. It is known that each technique has resulted in the death of cancer cells, with bio-effects attributed primarily to thermal actions and inertial cavitation. Nevertheless, protocols using ultrasounds and avoiding thermal draw backs are needed. Moreover, the application of these agents is important as it establishes a 
therapeutic-diagnostic platform for anti-cancer therapy [15]. In this scenario, we have set up a protocol to test LICUS effects on a PDAC, or pancreatic cancer tumor model. The latter was chosen with the ultimate goal to address the need of therapeutic approaches to treat this oncological malignancy. It is known that pancreatic cancer has a very high mortality rate, with an incidence that is increasing in economically developed countries, such as the USA, due to lifestyle habits [16]. We have found that the executed treatments are capable of avoiding thermal damage to the cells and that, according to literature, cell mortality can be ascribed only to LICUS effect.

Cell mortality was assessed using the BioFilmAnalyzer software, analyzing the spheroid images acquired with the confocal fluorescence microscope. We have found that the frequency capable of inducing cell death through LICUS treatment is $3 \mathrm{MHz}$.

The analysis of cell mortality allows for the definition that the $3 \mathrm{MHz}$ stimulation frequency is the most effective in inducing cancer cell death. With this set up, further frequencies and intensities will be investigated to optimize treatment times. Moreover, data obtained with the Annexin PI staining suggest that the $3 \mathrm{MHz}$ treatment frequency is capable of inducing cell death, which can be conceivably interpreted as a necroptosis process. This is evident from the plots in all three pancreatic cancer cell lines, where a strong increase in cells in the Q3 quadrant is evident. Such findings were also confirmed from the analysis of the brightfield images of spheroids, showing an enlarged shape of $3 \mathrm{MHz}$ treated cells, which is a sign suggesting the cell transition towards necroptosis. The high percentage of dead cells emerging from the Annexin PI analysis is justified because the staining allows for the quantification of dead cells in the external surface of the spheroids, while for apoptosis analysis, spheroids are disrupted, being able to also quantify dead cells in the central core of the spheroids. Such consideration holds true both for treated and control cells. Moreover, in the brightfield image cells in spheroids treated with $3 \mathrm{MHz}$ manifested a migratory behavior, which is consistent with the effect on proliferation and migration reported in literature and we truly believe it is directly due to the US treatment [9].

We believe we have obtained preliminary but significant results in LICUS treatment of pancreatic tumor spheroids with a single US treatment.

The setup and therapeutic protocol represent an innovative strategy for cancer treatment per se and we are confident that, after a proper validation, this can be useful to achieve at least $40 \%$ reduction in grafted tumor mass, comparable to that obtained with immunotherapeutic approaches tested in vivo with the administration of nanoparticles [17]. Despite the promising applications of the platform, some improvements will be mandatory, such as the test of the set up in a more complex 3D culture, which should encompass extracellular matrix components and the use of pancreatic stellate cells (PSC) [18]. Moreover, in vivo tests will be a further step in order to better clarify the biological effects of US treatment, after a proper adaptation of the ultrasound set up. The system will also be useful to allow for the testing of different effects between LICUS and LIPUS efficacy using the same frequencies and exposition time, both alone and in combination with the use of small molecules and antibody fragments, which allow precise tumor penetration and can be easily administered both for therapeutic and diagnostic purposes [12,19-21]. The latter opens the way for a combined pharmacological approach to PDAC treatment.

Author Contributions: Conceptualization, A.C., L.C. and A.A.; methodology, M.D., S.A., and C.D.; formal analysis M.D., S.A., C.D., J.I., L.M. and M.L.; investigation, M.D., S.A., C.D., M.R. and J.I.; data curation, M.D., S.A., C.D., M.R. and J.I.; writing-original draft preparation, M.D., S.A. and C.D.; writing-review and editing, M.D. and S.A.; supervision, A.C., L.C. and A.A.; project administration, A.C., L.C. and A.A.; funding acquisition, A.C., L.C. and A.A. All authors have read and agreed to the published version of the manuscript.

Funding: This research was funded by Associazione Italiana per la Ricerca sul Cancro (AIRC, Grant $\mathrm{N}^{\circ}$ IG 21510 to AA), by PRIN Italian Ministry of University and Research (MIUR) "Leveraging basic knowledge of ion channel network in cancer for innovative therapeutic strategies (LIONESS)" 20174TB8KW to AA and pHioniC: European Union's Horizon 2020 grant No 813834 to AA. Claudia 
Duranti was supported by an AIRC fellowship for Italy ID 24020. The work was also supported by Ente Cassa di Risparmio di Firenze to the TACTUS project for DINFO.

Institutional Review Board Statement: Not applicable.

Informed Consent Statement: Not applicable.

Data Availability Statement: The data presented in this study are available in the present article.

Conflicts of Interest: The authors declare no conflict of interest.

\section{References}

1. Steinhauser, M.O.; Schmidt, M. Destruction of cancer cells by laser-induced shock waves: Recent developments in experimental treatments and multiscale computer simulations. Soft Matter 2014, 10, 4778-4788. [CrossRef] [PubMed]

2. Morton, W.M.; Douglas, L.M.; Andrew, A.B. A review of in vitro bioeffects of inertial ultrasonic cavitation from a mechanistic perspective. Ultrasound Med. Biol. 1996, 22, 1131-1154.

3. Furusawa, Y.; Fujiwara, Y.; Campbell, P.; Zhao, Q.L.; Ogawa, R.; Hassan, M.A.; Tabuchi, Y.; Takasaki, I.; Takahashi, A.; Kond, T. DNA Double-Strand Breaks Induced by Cavitational Mechanical Effects of Ultrasound in Cancer Cell Lines. PLoS ONE 2012, 7, e29012. [CrossRef] [PubMed]

4. Jiang, X.; Savchenko, O.; Li, Y.; Qi, S.; Yang, T.; Zhang, W.; Chen, J. A Review of Low-Intensity Pulsed Ultrasound for Therapeutic Applications. IEEE Transect. Biomed. Eng. 2019, 10, 2704-2718. [CrossRef] [PubMed]

5. Martin, E. The Cellular Bioeffects of Low Intensity Ultrasound. Ultrasound 2009, 17, 214-219. [CrossRef]

6. Fontes-Pereira, A.J.; Teixeira, R.C.; de Oliveira, A.J.B.; Pontes, R.W.F.; de Barros, R.S.M.; Negrão, J.N.C. The effect of low-intensity therapeutic ultrasound in induced fracture of rat tibiae. Braz. Orthop. Acta 2013, 1, 18-22. [CrossRef] [PubMed]

7. Zhao, Y.; Xing, J.; Xing, J.Z.; Ang, W.T.; Chen, J. Application of low-intensity pulsed ultrasound to increase monoclonal antibody production in $\mathrm{CHO}$ cells using shake flasks or wavebags. Ultrasonic 2014, 54, 1439-1447. [CrossRef] [PubMed]

8. Huang, D.; Gao, Y.; Wang, S.; Zhang, W.; Cao, H.; Zheng, L.; Chen, Y.; Zhang, S.; Chen, J. Impact of low-intensity pulsed ultrasound on transcription and metabolite compositions in proliferation and functionalization of human adipose-derived mesenchymal stromal cells. Sci. Rep. 2020, 10, 13690. [CrossRef] [PubMed]

9. Sengupta, S.; Balla, V.K. A review on the use of magnetic fields and ultrasound for non-invasive cancer treatment. J. Adv. Res. 2018, 14, 97-111. [CrossRef] [PubMed]

10. Sung, H.; Ferlay, J.; Siegel, R.L.; Laversanne, M.; Soerjomataram, I.; Jemal, A.; Bray, F. Global cancer statistics 2020, GLOBOCAN estimates of incidence and mortality worldwide for 36 cancers in 185 countries. CA Cancer J. Clin. 2021, 71, 209-249. [CrossRef] [PubMed]

11. Capineri, L. A $15 \mathrm{MHz}$ bandwidth, $60 \mathrm{~V}-\mathrm{pp}$, low distortion power amplifier for driving high power piezoelectric transducers. Rev. Sci. Instrum. 2014, 85, 104701. [CrossRef] [PubMed]

12. Duranti, C.; Iorio, J.; Lottini, T.; Lastraioli, E.; Crescioli, S.; Bagni, G.; Lulli, M.; Capitani, C.; Bouazzi, R.; Stefanini, M.; et al Harnessing the hERG1/ $\beta 1$ Integrin Complex via a Novel Bispecific Single-chain Antibody: An Effective Strategy against Solid Cancers. Mol. Cancer Ther. 2021, 20, 1338-1349. [CrossRef] [PubMed]

13. Patra, B.; Peng, C.C.; Liao, W.H.; Lee, C.H.; Tung, Y.C. Drug testing and flow cytometry analysis on a large number of uniform sized tumor spheroids using a microfluidic device. Sci. Rep. 2016, 6, 21061. [CrossRef] [PubMed]

14. Beer, M.; Kuppalu, N.; Stefanini, M. A novel microfluidic 3D platform for culturing pancreatic ductal adenocarcinoma cells: Comparison with in vitro cultures and in vivo xenografts. Sci. Rep. 2017, 7, 1325. [CrossRef] [PubMed]

15. Webster, J.G. The Measurement, Instrumentation, and Sensors; CRC Press: Chicago, IL, USA, 1999.

16. Bloomfield, P.E. Experimental study of the aucustical properties of polymers utilized to construct PVDF Ultrasonic Transducers and the Acousto-Electric Properties of PVDF and P(VDF/TrFE) Films. IEEE Transect. Ultrason. Ferroelectr. Freq. Control 2000, 6, 47.

17. Bogachev, M.I.; Volkov, V.Y.; Markelov, O.A.; Trizna, E.Y.; Baydamshina, D.R.; Melnikov, V.; Murtazina, R.R.; Zelenikhin, P.V.; Sharafutdinov, I.S.; Kayumov, A.R. Fast and simple tool for the quantification of biofilm-embedded cells sub-populations from fluorescent microscopic images. PLoS ONE 2018, 5, e0193267. [CrossRef] [PubMed]

18. Manoli, S.; Coppola, S.; Duranti, C.; Lulli, M.; Magni, L.; Kuppalu, N.; Nielsen, N.; Schmidt, T.; Schwab, A.; Becchetti, A.; et al. The Activity of Kv 11.1 Potassium Channel Modulates F-Actin Organization During Cell Migration of Pancreatic Ductal Adenocarcinoma Cells. Cancers 2019, 11, 135. [CrossRef] [PubMed]

19. Wood, A.K.; Sehgal, C.M. A review of low-intensity ul-trasound for cancer therapy. Ultrasound Med. Biol. 2015, 41, 905-928. [CrossRef] [PubMed]

20. Duranti, C.; Lastraioli, E.; Iorio, J.; Capitani, C.; Carraresi, L.; Gonnelli, L.; Arcangeli, A. Expression and purification of a novel single-chain diabody (scDb-hERG1/ $\beta 1$ ) from Pichia pastoris transformants. Protein Expr. Purif. 2021, 184, 105879. [CrossRef] [PubMed]

21. Duranti, C.; Carraresi, L.; Sette, A.; Stefanini, M.; Lottini, T.; Crescioli, S.; Crociani, O.; Iamele, L.; De Jonge, H.; Gherardi, E.; et al. Generation and characterization of novel recombinant anti-hERG1 scFv antibodies for cancer molecular imaging. Oncotarget 2018, 9, 34972-34989. [CrossRef] [PubMed] 